\title{
BMJ Open Value of high-sensitivity C-reactive protein assays in predicting atrial fibrillation recurrence: a systematic review and meta-analysis
}

\author{
Chia-Hung Yo, ${ }^{1}$ Si-Huei Lee, ${ }^{2}$ Shy-Shin Chang, ${ }^{3,4}$ Matthew Chien-Hung Lee, ${ }^{5}$ \\ Chien-Chang Lee $6,7,8$
}

To cite: Yo C-H, Lee S-H, Chang S-S, et al. Value of high-sensitivity C-reactive protein assays in predicting atrial fibrillation recurrence: a systematic review and meta-analysis. BMJ Open 2014;4:e004418.

doi:10.1136/bmjopen-2013004418

- Prepublication history and additional material for this paper is available online. To view these files please visit the journal online (http://dx.doi.org/10.1136/ bmjopen-2013-004418).

Received 7 November 2013 Revised 29 January 2014 Accepted 31 January 2014

CrossMark

For numbered affiliations see end of article.

Correspondence to Dr Chien-Chang Lee; cclee100@gmail.com

\section{ABSTRACT}

Objectives: We performed a systematic review and meta-analysis of studies on high-sensitivity C-reactive protein (hs-CRP) assays to see whether these tests are predictive of atrial fibrillation (AF) recurrence after cardioversion.

Design: Systematic review and meta-analysis. Data sources: PubMed, EMBASE and Cochrane databases as well as a hand search of the reference lists in the retrieved articles from inception to December 2013.

Study eligibility criteria: This review selected observational studies in which the measurements of serum CRP were used to predict AF recurrence. An hsCRP assay was defined as any CRP test capable of measuring serum CRP to below $0.6 \mathrm{mg} / \mathrm{dL}$.

Primary and secondary outcome measures: We summarised test performance characteristics with the use of forest plots, hierarchical summary receiver operating characteristic curves and bivariate random effects models. Meta-regression analysis was performed to explore the source of heterogeneity.

Results: We included nine qualifying studies comprising a total of 347 patients with AF recurrence and 335 controls. A CRP level higher than the optimal cut-off point was an independent predictor of $A F$ recurrence after cardioversion (summary adjusted OR: 3.33; 95\% Cl 2.10 to 5.28 ). The estimated pooled sensitivity and specificity for hs-CRP was $71.0 \%(95 \% \mathrm{Cl} 63 \%$ to $78 \%$ ) and $72.0 \%$ ( $61 \%$ to $81 \%$ ), respectively. Most studies used a CRP cut-off point of $1.9 \mathrm{mg} / \mathrm{L}$ to predict long-term AF recurrence ( $77 \%$ sensitivity, $65 \%$ specificity), and $3 \mathrm{mg} / \mathrm{L}$ to predict short-term AF recurrence ( $73 \%$ sensitivity, $71 \%$ specificity).

Conclusions: hs-CRP assays are moderately accurate in predicting AF recurrence after successful cardioversion.

\section{INTRODUCTION}

Atrial fibrillation (AF) is the most common arrhythmia in clinical practice, although the prevalence is highest among people of advanced age. ${ }^{1} \quad 2$ AF poses a significant

\section{Strengths and limitations of this study}

- This meta-analysis finding supports that measurement of C-reactive protein (CRP) levels before cardioversion can aid in the prediction of atrial fibrillation recurrence.

- We reported summary likelihood ratios as an ancillary measure of predictive accuracy.

- We used a bivariate random effect model to account for the inherent negative correlation arising from different cut-off values used in different studies, and occurring between the logit true positive rates and false positive rates.

- Results of sensitivity analysis did not show a significantly different overall predictive accuracy between long-term and short-term follow-up, however a heterogeneity tended towards betweenstudy variability.

- Current summary estimates based on the one cut-off point may thus have under-evaluated the clinical usefulness of hs-CRP assays.

economic burden, with a $66 \%$ increase in hospital admissions over the past two decades. It is estimated that the number of patients affected by $\mathrm{AF}$ is likely to grow 2.5-fold by the year $2050 .^{1-3}$ In addition, AF may lead to debilitating complications such as ischaemic stroke and heart failure. Although ventricular rate control is an acceptable treatment strategy in many patients, some patients may remain symptomatic despite adequate rate controls. For this group of patients, cardioversion may be the treatment of choice. Electrical cardioversion can restore sinus rhythm effectively in most patients and can act with antiarrhythmic drugs synergistically to enhance the cardioversion success rate. ${ }^{4}$ However, cardioversion is not the definite treatment. Approximately $50 \%$ of patients undergoing cardioversion usually present with recurrence of $\mathrm{AF}$ within 3-6 months of cardioversion despite ongoing antiarrhythmic treatment. ${ }^{5}$ Left ventricular 
dysfunction, left atrial enlargement, arrhythmia duration and history of hypertension are major risk factors for $\mathrm{AF}$ recurrence. ${ }^{6}$ However, recent studies have indicated that inflammation, necrosis and fibrosis play roles in the structural remodelling process of the atria, contributing to the perpetuation or recurrence of $\mathrm{AF}$.

C-reactive protein (CRP) is an acute-phase reactant whose levels increase in response to proinflammatory cytokines, notably interleukin- 6 , and other endogenous signals of innate immunity or tissue damage. CRP has recently been shown to be associated with cardiovascular risk and $\mathrm{AF}$ recurrence. A previous meta-analysis revealed that CRP is elevated in patients with $\mathrm{AF}^{7}$ However, five of the six studies included in that analysis used traditional automated immunonephelometric assays to measure CRP. Unfortunately, those assays are insufficiently sensitive for measuring the low level of inflammation associated with AF. A newer enzyme immunoassay, namely high-sensitivity CRP (hs-CRP), is capable of measuring serum CRP below $0.6 \mathrm{mg} / \mathrm{dL}$ and may further enhance the predictability of AF recurrence. ${ }^{8}$ Since 2006, several studies evaluating the accuracy of hs-CRP in predicting AF recurrence have been published, ${ }^{9-19}$ warranting a systemic and quantitative summary of current evidence on the accuracy of CRP in predicting AF recurrence after cardioversion.

\section{METHODS}

Identification of studies

General bibliographic databases (MEDLINE and EMBASE) were searched from inception to April 2013. The medical subject heading $(\mathrm{MeSH})$ and text words for the term C-reactive protein were combined with the MeSH term 'diagnosis of atrial fibrillation'. The search was limited to human studies with no language restrictions. In addition to the electronic search, reference lists in all known reviews and primary studies were checked manually.

\section{Selection criteria}

This review focused on observational studies in which the measurements of serum CRP were used to predict $\mathrm{AF}$ recurrence. The population of interest comprised patients with paroxysmal or persistent AF who underwent electric cardioversion, ablation therapy or pharmacological cardioversion. AF recurrence was defined as $\mathrm{AF}$ documented by ECG at any time after the cardioversion during the follow-up period. Generally, patients were instructed to return to the clinic if the symptoms such as palpitations, shortness of breath or chest discomfort developed after cardioversion. We included studies using a cohort design or case-control design with appropriate controls. Two reviewers independently assessed eligible articles for inclusion. Disagreements were initially resolved by consensus and using arbitration by a third reviewer if consensus could not be reached by the two reviewers. We extracted data from the included studies. Data collected include study design, participants, country, period of recruitment, hs-CRP assay, cut-off points, length of follow-up period and recurrence of AF. One reviewer extracted the data and a second reviewer independently verified the correctness of the extracted data.

\section{Quality assessment}

We assessed the methodological quality of the selected studies using a well validated tool for assessment of quality of diagnostic accuracy studies (Quality Assessment of Diagnostic Accuracy Studies, QUADAS). ${ }^{20}$ The QUADAS instrument scrutinises characteristics of study designs, population, index tests and reference standards that may be associated with risk of bias. These features included the spectrum of patients, whether index tests and reference standards were evaluated and interpreted independently to avoid incorporation bias, and whether all patients underwent the same reference standards to avoid differential or partial verification bias.

\section{Data abstraction}

One reviewer independently extracted the data and a second reviewer independently verified the data. Extracted data comprised the following: overall study characteristics (including the first author, country, language and date of publication); patient characteristics (including age range and pre-existing $\mathrm{AF}$ ); quantitative data required for construction of a $2 \times 2$ table (including number of participants, sensitivity, specificity and recurrence case number; see online supplementary table); information regarding the hs-CRP assay (including brand name of the test kit, cut-off levels and quantitative or semi-quantitative nature of the test); and study settings. In studies that reported multiple pairs of sensitivity and specificity data, we consistently used the data with the highest Youden index (sensitivity+specificity-1) and performed a sensitivity analysis at a later stage.

\section{Quantitative data synthesis}

We performed a meta-analysis of diagnostic test accuracy of CRP testing for the prediction of recurrent AF. We calculated the pooled sensitivity and specificity, positive and negative likelihood ratios, and the diagnostic OR of CRP, along with the respective 95\% CIs, using a bivariate meta-analysis model. ${ }^{21}$ Likelihood ratios were then translated to post-test probability by use of Fagan's plot. We constructed a hierarchical summary receiver operating characteristic curve that plots sensitivity versus specificity and calculated the area under the curve. ${ }^{22}$ We evaluated the degree of between-study heterogeneity by using the $\mathrm{I}^{2}$ test. ${ }^{23}$ To explore the clinical sources of heterogeneity, we defined the potential explanatory variables a priori and performed subgroup analysis to see if the accuracy estimates changed significantly across various subgroups. The presence and the effect of publication bias were examined using a combination of the Egger tests. ${ }^{24}$ Statistical analyses were conducted using STATA V.11.0, notably with the user-written 'midas' and 'metandi' 
programs. All statistical tests were two-sided and statistical significance was defined as $\mathrm{p}<0.05$.

\section{Search results and study characteristics}

The flow of inclusion and exclusion is summarised in figure 1. Using our search criteria, we identified 784 studies, of which 352 were from PubMed and 432 were from EMBASE. A total of 752 citations were excluded based on predefined criteria. No additional citations were identified from the reference lists. A total of 32 articles were retrieved for full-text review, and 23 were excluded due to various reasons detailed in figure 1 . A total of nine studies that evaluated the accuracy of hs-CRP tests in predicting AF recurrence after cardioversion were finally included in the meta-analysis. The nine studies included a total of 682 patients with AF after successful cardioversion, of which 347 (50.9\%) developed recurrence.

\section{Characteristics of included studies}

Table 1 lists the study and population characteristics of the nine patient populations, excluding five studies that do not have sufficient data for statistical analysis. ${ }^{25-29}$ The mean age of patients in the included studies ranged from 55.1 to 67.9 years and the mean follow-up period ranged from 30 days to 1 year. Seven studies included patients with persistent AF, while two studies included patients with paroxysmal AF. Seven studies used electric shock, one used circumferential pulmonary vein isolation (also known as electric ablation) and the other used intravenous amiodarone as the primary method for cardioversion. A total of seven studies provided multivariate (adjusted) ORs to evaluate the independent predictive value of CRP levels. These studies generally adjusted for potential predictors of AF recurrence such as age, sex, use of antiarrhythmic agents, and heart structural and functional parameters. All studies showed that CRP was a significant independent predictor of $\mathrm{AF}$ recurrence. Associated adjusted ratios and adjusted variables are summarised in table 1 .

\section{Quality assessment}

Results of the quality assessment of studies of diagnostic accuracy are summarised in figure 2. All studies were prospective and enrolled consecutive outpatients with AF after cardioversion. Three studies had a short follow-up period (ie, $\leq 0.5$ or 1 year). Although most of the studies did not indicate whether physicians were blinded to the index tests when diagnosing AF recurrence, the determination of $\mathrm{AF}$ recurrence was not affected by the knowledge of hs-CRP test results and risk of incorporation bias was minimal. None of the studies reported the undetermined results or withdrawals.
Figure 1 A simplified flow chart to identify and to include studies. Among 752 citations in MEDLINE and EMBASE from inception to December 2013, a search limited to human studies using 'C-reactive protein' and the medical subject heading (MeSH) term 'diagnosis of atrial fibrillation' resulted in 32 potentially relevant articles for further review. After careful scrutinisation on full text, nine articles were left for meta-analysis.

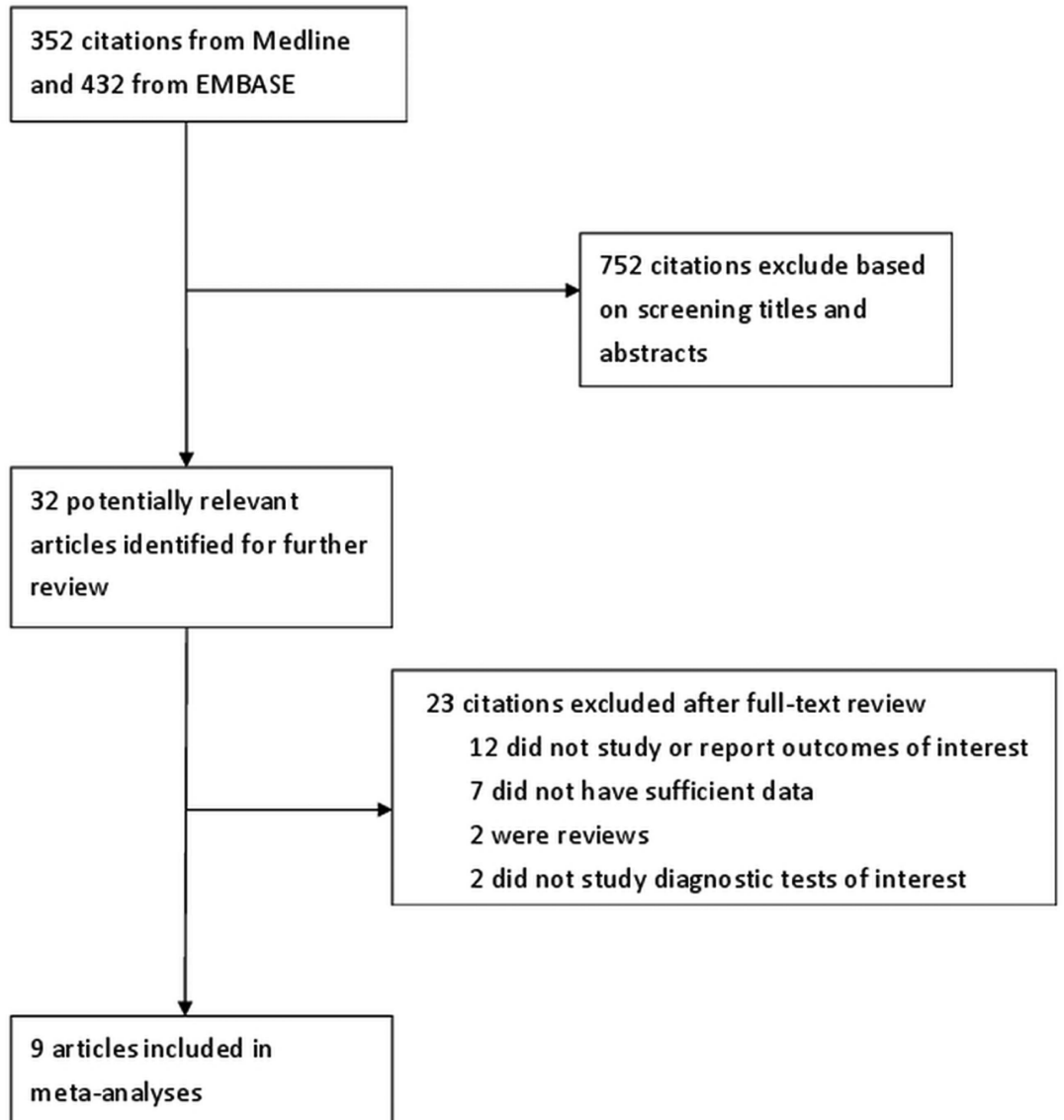


Table 1 Summary of the characteristics of the included studies

\begin{tabular}{|c|c|c|c|c|c|c|c|c|c|}
\hline $\begin{array}{l}\text { Author, year, } \\
\text { country }\end{array}$ & $\begin{array}{l}\text { Mean } \\
\text { age }\end{array}$ & $\begin{array}{l}\text { Prevalence } \\
\text { (N) }\end{array}$ & $\begin{array}{l}\text { Follow-up } \\
\text { time }\end{array}$ & $\begin{array}{l}\text { Cut-off } \\
\text { (mg/L) }\end{array}$ & AF type & Cardioversion & $\begin{array}{l}\text { Sensitivity, } \\
\text { pecificity (\%) }\end{array}$ & Adjusted OR & Adjusted variables \\
\hline $\begin{array}{l}\text { Wazni, 2005, } \\
\text { USA }^{16}\end{array}$ & 67.3 & $0.68(111)$ & 76 days & 3.1 & Persistent AF & Electric & 59,69 & 2.0 (1.2 to 3.2$)$ & $\begin{array}{l}\text { Age, sex, duration of } A F \text {, coronary } \\
\text { artery disease, hypertension, left } \\
\text { ventricular hypertrophy, LAD }\end{array}$ \\
\hline $\begin{array}{l}\text { Zarauza, 2006, } \\
\text { Spain }^{19}\end{array}$ & 62.7 & $0.43(37)$ & 30 days & 3.0 & Persistent AF & Electric & 81,67 & $3.7(1.3$ to 10.8$)$ & $\begin{array}{l}\text { Sex, age, time, size of left atrium, } \\
\text { history of hypertension, } \\
\text { pharmacological treatment }\end{array}$ \\
\hline $\begin{array}{l}\text { Watanabe, 2006, } \\
\text { Japan }^{18}\end{array}$ & 64 & $0.76(84)$ & 1 year & 0.6 & Persistent AF & Electric & 75,90 & $5.3(2.5$ to 11.5$)$ & $\begin{array}{l}\text { Sex, coronary artery disease, } \\
\text { hypertension, smoking, diabetes, AF } \\
\text { duration, LAD, LVEF }\end{array}$ \\
\hline $\begin{array}{l}\text { Loricchio, 2007, } \\
\text { Italy }^{15}\end{array}$ & 67 & $0.52(102)$ & 1 year & 1.9 & Persistent AF & Electric & 87,37 & $5.0(1.8$ to 14.3$)$ & $\begin{array}{l}\text { Age, gender, EF, LAD, } \\
\text { hypertension, diabetes, } \\
\text { pharmacological treatment }\end{array}$ \\
\hline $\begin{array}{l}\text { Lombardi, 2008, } \\
\text { Italy }^{14}\end{array}$ & 67 & $0.34(53)$ & 21 days & 3.6 & Persistent AF & Electric & 64,83 & 1.6 (1.0 to 2.5$)$ & $\begin{array}{l}\text { Age, LAD, LAA, LAAEV, NTproBNP } \\
\text { level, history of AF, AF duration, } \\
\text { pharmacological treatment }\end{array}$ \\
\hline $\begin{array}{l}\text { Henningsen, } \\
\text { 2009, Denmark }{ }^{12}\end{array}$ & 65 & $0.68(56)$ & 180 days & 3.0 & Persistent AF & Electric & 60,83 & $7.7(1.9 \text { to } 31.1)^{*}$ & NA \\
\hline $\begin{array}{l}\text { Rizos, } 2010 \text {, } \\
\text { Greece }^{17}\end{array}$ & 67.9 & $0.64(61)$ & 1 year & 2.3 & Paroxysmal AF & Pharmacologic & 72,68 & $6.2(2.2$ to 17.6$)$ & $\begin{array}{l}\text { IL-6, age, gender, PAF history, LAD, } \\
\text { EF, diabetes, smoking }\end{array}$ \\
\hline $\begin{array}{l}\text { Liu, 2011, } \\
\text { China }^{13}\end{array}$ & 55.1 & $0.39(44)$ & 1 year & 1.9 & Paroxysmal AF & $\begin{array}{l}\text { Electric } \\
\text { ablation }\end{array}$ & 79,70 & $5.1(2.1$ to 12.1$)$ & $\begin{array}{l}\text { Age, gender, type of } A F \text {, duration of } \\
A F, L A D, L V E F \text {, plasma hs-CRP } \\
\text { concentration }\end{array}$ \\
\hline $\begin{array}{l}\text { Barassi, } 2012 \\
\text { Italy }^{10}\end{array}$ & 66.9 & $0.33(57)$ & 21 days & 3.0 & Persistent AF & Electric & 74,84 & $14.9(3.9 \text { to } 57.2)^{*}$ & NA \\
\hline
\end{tabular}

Italy ${ }^{10}$

“Crude effect estimate.

$\mathrm{AF}$, atrial fibrillation; BNP, b-type natriuretic peptide; EF, ejection fraction; hs-CRP, high-sensitivity C-reactive protein; IL, interleukin; LAA, left atrial appendage; LAAEF, left atrial appendage

ejection fraction; LAD, left anterior descending coronary artery; LVEF, left ventricular ejection fraction; NA, not applicable; NTproBNP, N-terminal prohormone of brain natriuretic peptide; PAF,

paroxysmal atrial fibrillation. 
Figure 2 The quality assessment of diagnostic accuracy on studies. A spectrum of features were analysed to avoid bias using a well validated tool, Quality Assessment of Diagnostics Accuracy Studies (QUADAS). Percentage for each feature was independently evaluated among the studies. It is worthy of attention that none of the studies explained the withdrawal and reported undetermined results, likely to compromise the quality of diagnostic accuracy.

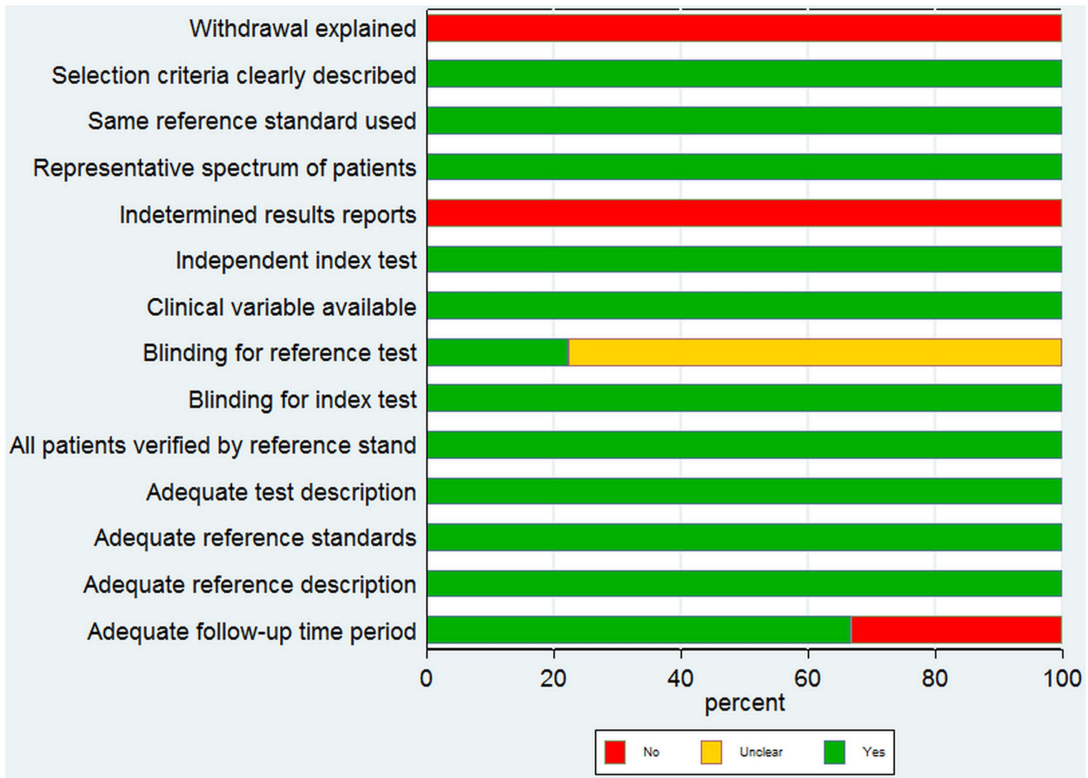

\section{Diagnostic accuracy indices}

Sensitivity, specificity and diagnostic OR

The estimated sensitivity and specificity were relatively consistent across studies $\left(\mathrm{I}^{2}=14.6 \%\right)$. Table 2 shows the results of individual and combined sensitivity estimates for the tests. The estimated pooled sensitivity and specificity for hs-CRP were $71.0 \%$ (95\% CI $63 \%$ to $78 \%$ ) and $72.0 \%$ ( $61 \%$ to $81 \%$ ), respectively. The pooled prevalence of $\mathrm{AF}$ recurrence was $54 \%$ herein, and we used it as the pre-test probability. With a pooled positive likelihood ratio of 2.57 and a negative likelihood ratio of 0.4 , the post-test probability for $\mathrm{AF}$ recurrence for a positive hs-CRP test result was $72 \%$ and a post-test probability for a negative hs-CRP test result was 29\%. The area under the receiver operating curve showed an acceptable overall measurement of discrimination (0.77, figure 3 ). Figure 4 shows the forest plot of the ORs.

\section{Subgroup analysis and meta-regression}

In view of the potential influence of spectrum variability, we considered the duration of follow-up, mode of cardioversion and type of $\mathrm{AF}$ in the study patients to be important. hs-CRP test results generally had higher sensitivity and lower specificity in predicting long-term over short-term AF recurrence. Excluding two studies not using electric shock as the primary cardioversion method did not significantly alter the predictive accuracy. Similarly, focusing the study patients on persistent AF population had similar results as compared with the main overall analysis. Exploratory meta-regression analysis did not find that any pre-specified covariate significantly changed the effect estimate.

\section{DISCUSSION}

This meta-analysis shows that elevated CRP levels are independently predictive of $\mathrm{AF}$ recurrence in patients with persistent or paroxysmal AF who have undergone successful cardioversion. This finding supports that measurement of CRP levels before cardioversion can aid in the prediction of AF recurrence. Despite the modest pooled sensitivity and specificity, the rule-in diagnostic value was still high, given the high recurrence rate of $\mathrm{AF}$ observed in these included studies. A positive hs-CRP test result at baseline can predict a $73 \%$ chance of AF recurrence in the 6-12 months following cardioversion.

Previous studies have examined risk factors that predict AF recurrence. Traditional clinical risk factors for recurrence include history of multiple AF episodes, use of diuretic treatment, higher CHADS-2 (congestive heart failure, history of hypertension, age $\geq 75$ years, diabetes mellitus, and past history of stroke or transient ischaemic attack doubled) index score, and frequent use of amiodarone, calcium-channel blockers, class 1C drugs and digitalis. ${ }^{30}$ Although each of these factors could predict $\mathrm{AF}$ recurrence with some accuracy, a quantitative combination of these predictors is not available, and the clinical utility of these variables remains questionable. This also suggests that a multivariate prediction model should be developed for AF recurrence, and that hs-CRP should be a candidate for inclusion in the model.

During the past decade, serum biomarkers have emerged as practical tools to help in the early identification of patients at high risk for various cardiac events. Elevation of inflammatory markers is associated with sudden cardiac death in patients with heart failure or coronary artery disease, and onset of ventricular arrhythmia. $^{32-35}$ Of note, there is abundant evidence that elevated serum levels of CRP are associated with the genesis and perpetuation of AF. CRP is the most commonly used clinical inflammatory biomarker. It is mainly produced in liver and by inflammatory cells in 


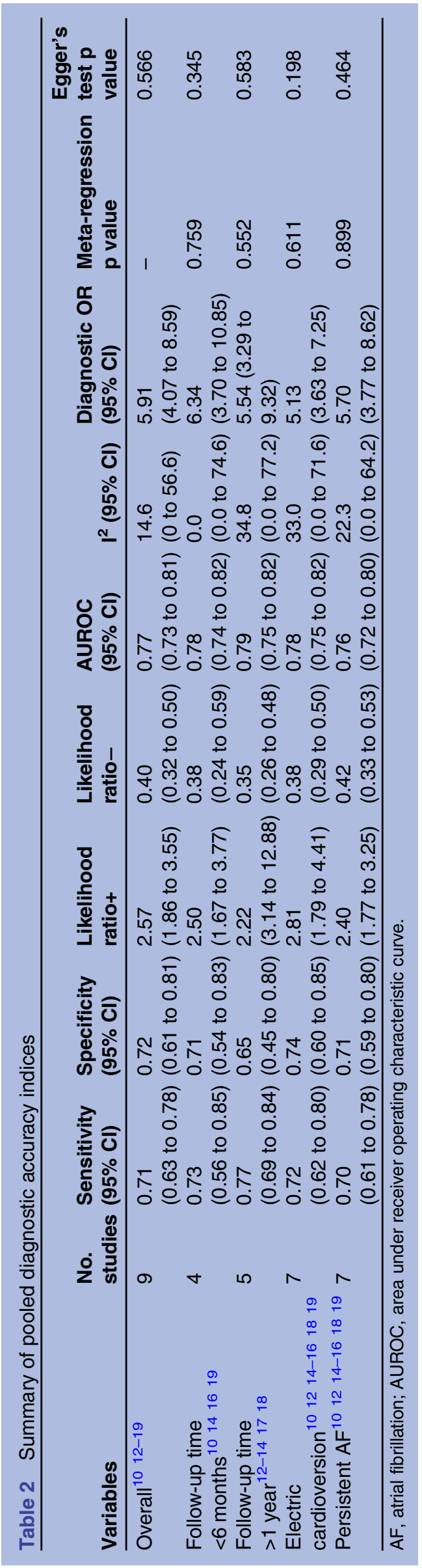

response to proinflammatory cytokine stimulation. Although the pathophysiology of AF remains elusive, there is pathophysiological evidence supporting the role of inflammation in the initiation, maintenance and perpetuation of $\mathrm{AF}^{36}$ Clinically, $\mathrm{AF}$ is frequently associated with local inflammatory diseases such as myocarditis or pericarditis, and systemic inflammatory status, such as postoperative state and severe sepsis. Histologically, structural remodelling of the atria manifested by loss of myocardium and increased atrial fibrosis is a hallmark of $\mathrm{AF}^{37}$ Inflammatory cell infiltrates and oxidative damage have been demonstrated in atrial biopsy specimens from AF patients. ${ }^{38}$ Activated inflammatory cells in conjunction with reactive oxygen species, cytokines and growth factors, may ultimately lead to matrix deposition with atrial fibrosis. Recent evidence also shows that the uses of immune-modulating agents such as statins, ACE inhibitors or glucocorticoids modulate the course of $\mathrm{AF}^{39}$

In addition to CRP, b-type natriuretic peptide (BNP) has been shown to be an indicator of new onset $\mathrm{AF}$ and $\mathrm{AF}$ recurrence after successful cardioversion. ${ }^{34} 35{ }^{40} \mathrm{BNP}$ is also produced in response to atrial pressure and volume overload and there is evidence that BNP is secreted by the atrium in patients with AF. A previous meta-analysis showed that the standardised mean difference in plasma BNP level between patients with nonrecurrence and patients with recurrence was -1.35 (95\% CI -2.17 to -0.53$).{ }^{41}$ Data on sensitivity and specificity in that study were not available. The comparative accuracy between BNP and hs-CRP in predicting AF recurrence thus requires further analysis.

There are both strengths and limitations in our study. Considering the limitation of sensitivity and specificity in clinical interpretation, we reported summary likelihood ratios (LRs) as an ancillary measure of predictive accuracy. The LRs indicate how much a given CRP testing result increases or decreases the probability of recurrence of AF. Post-test probabilities can be derived from pre-test probabilities and LRs, which are an important clinical parameter for major clinical decision making. Second, we used a bivariate random effect model to account for the inherent negative correlation arising from different cut-off values used in different studies, and occurring between the logit true positive rates and false positive rate. Third, we performed sensitivity analysis by restricting analysis within two broad categories of follow-up duration. Results of sensitivity analysis did not show a significantly different overall predicative accuracy between long-term and short-term prediction of AF recurrence. Nonetheless, it is noteworthy that the sensitivity may be overestimated in our study under the hypothesis where the inflammation may be symptomatic since none of the studies provided withdrawal and undetermined results, and the ascertainment of $\mathrm{AF}$ was passive. This event further introduces the differential verification bias. Moreover, our meta-analysis is restricted to distinguishing hs-CRP levels 


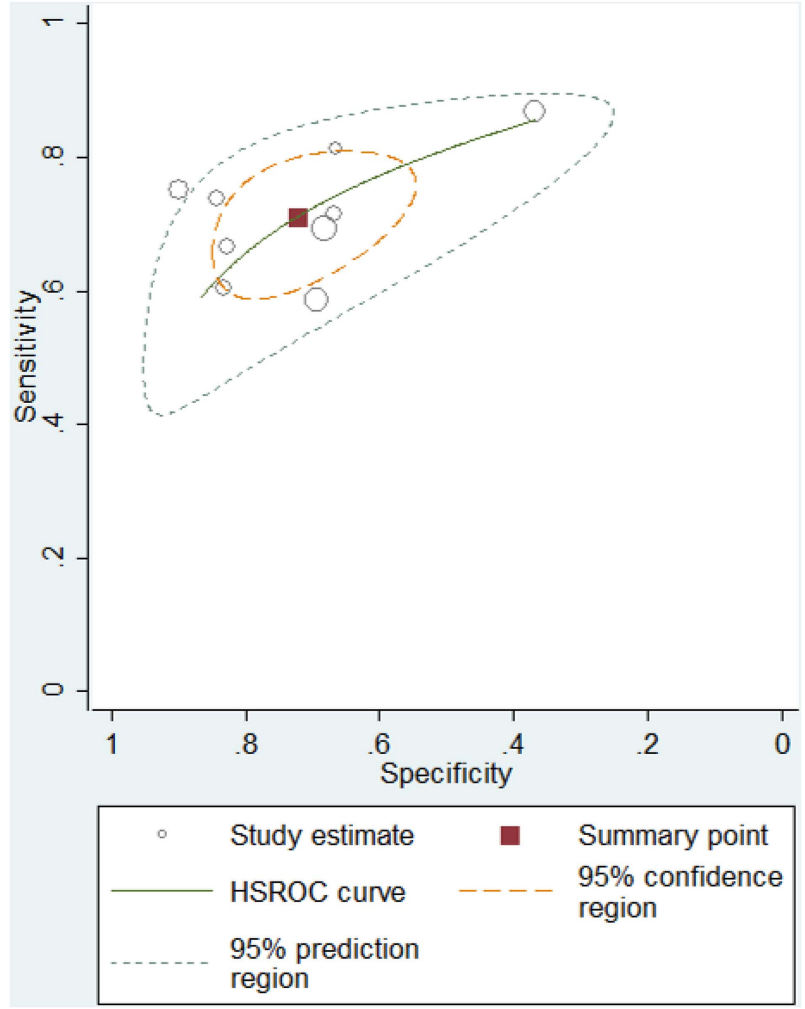

Figure 3 The receiver operating characteristic (ROC) curve of high-sensitivity $\mathrm{C}$-reactive protein (hs-CRP). Our analysis suggests it is highly possible to predict atrial fibrillation using C-creative protein since the area under the curve generates a measurement of discrimination $\sim 0.77$. The overall sensitivity and specificity are relatively high. Five out of nine studies fall in the $95 \% \mathrm{Cl}$ region, and eight out of nine in the $95 \%$ prediction region.

above or below $0.6 \mathrm{mg} / \mathrm{dL}$ because the authors in only one of the studies claimed to possess such capability. Finally, due to the lack of individual data, it is hard to determine whether the area under the receiver operating curve can be improved by the new assay either on overall or on individual studies. In general, potential sources of between-study variability included differences in incidence of $\mathrm{AF}$ recurrence, different threshold values of CRP concentration used and different duration for follow-up. Another limitation was the strategy we used to determine the optimal cut-off value. Most studies determined an optimal cut-off value to maximise both sensitivity and specificity. Although a single cut-off value is straightforward in clinical interpretation, it may make a marker neither sensitive nor specific enough to rule out or rule in an outcome of interest. A two cut-off value strategy, with one using a lower cut-off value to optimise the sensitivity (rule-out value) and the other using a higher cut-off value to optimise the specificity (rule-in value), would make better use of the information that a biomarker with a continuous value could provide. Current summary estimates based on the one cut-off point may thus have under-evaluated the clinical usefulness of hs-CRP assays. To make the best use of the biomarker information by adopting a two cut-off point strategy or a multi-cut-off point risk classification strategy, an individual data meta-analysis would be needed to overcome the limitations of this aggregated data meta-analysis.

\section{CONCLUSIONS}

Baseline CRP levels before cardioversion can independently predict $\mathrm{AF}$ recurrence after successful cardioversion. Given the high recurrence rate reported in most series, the modest positive likelihood ratio for hs-CRP assays still has high positive predictive value. Future studies should focus on the evaluation of two or multiple cut-off points. In the interim, their inclusion in existing pre-cardioversion evaluation algorithms should be considered.

Figure 4 Forest plot of the ORs.

Our study indicates that

high-sensitivity C-reactive protein

(hs-CRP) positive patients are

$\sim 5.91$ times more likely to develop a recurrence of atrial fibrillation than are hs-CRP negative patients. The estimated sensitivity and specificity were relatively consistent across studies $\left(I^{2}=14.6 \%\right)$.

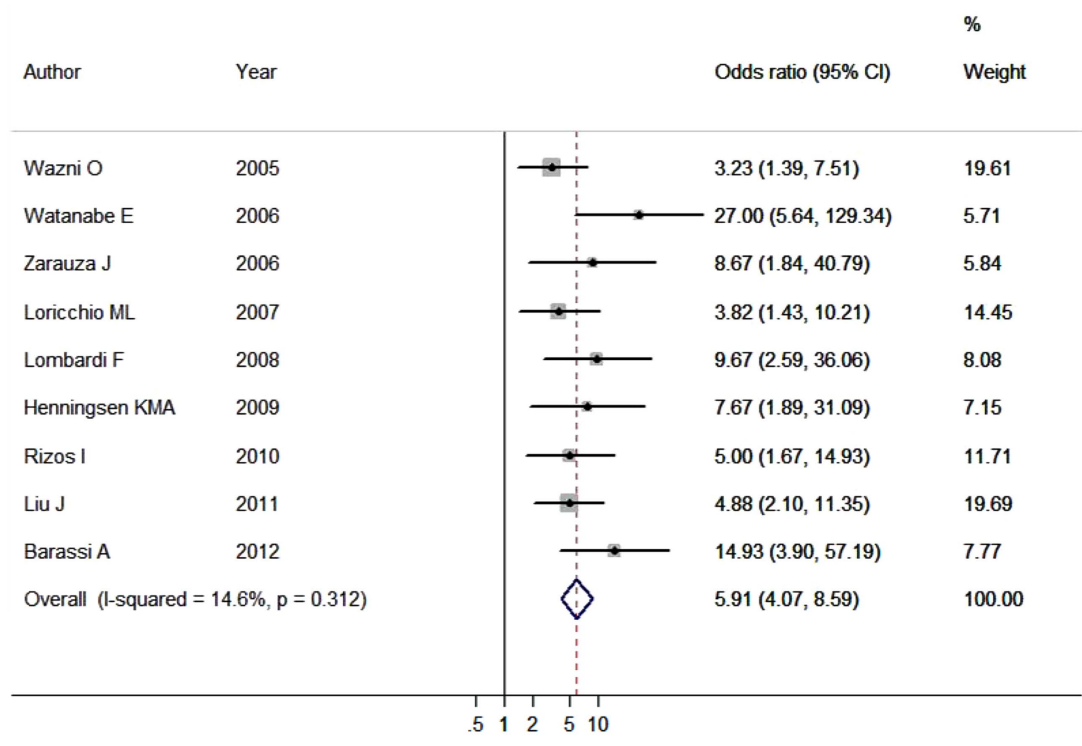


Author affiliations

${ }^{1}$ Department of Emergency Medicine, Far Eastern Memorial Hospital, New Taipei City, Taiwan

${ }^{2}$ Department of Rehabilitation and Physical Medicine, Taipei Veteran General Hospital, Taipei, Taiwan

${ }^{3}$ Department of Family Medicine, Chang Gung Memorial Hospital, Taoyuan, Taiwan

${ }^{4}$ Graduate Institute of Clinical Medical Sciences, College of Medicine, Chang Gung University, Taoyuan, Taiwan

${ }^{5}$ Medical Wisdom Consultants, Houston, Texas, USA

${ }^{6}$ Department of Epidemiology, Harvard School of Public Health, Boston, Massachusetts, USA

${ }^{7}$ Department of Emergency Medicine, National Taiwan University Hospital Yunlin Branch, Douliou, Taiwan

${ }^{8}$ Department of Emergency Medicine, National Taiwan University Hospital, Taipei, Taiwan

Contributors C-HY: study design, data management, statistics, first draft, fina draft and approval; S-HL: study design, scientific and statistic advisory, study monitoring, final draft and approval; S-SC: statistics, final draft and approval; MC-HL; data collection, final draft and approval; C-CL: design, scientific and statistic advisory, final draft and approval.

Funding This study was supported by grants of the Far Eastern Memorial Hospital, Taiwan (FEMH-2013_D_036).

Competing interests None.

Provenance and peer review Not commissioned; externally peer reviewed.

Data sharing statement The study results will be published in a peer-reviewed scientific journal. The extracted data will open to sharing upon request.

Open Access This is an Open Access article distributed in accordance with the Creative Commons Attribution Non Commercial (CC BY-NC 3.0) license, which permits others to distribute, remix, adapt, build upon this work noncommercially, and license their derivative works on different terms, provided the original work is properly cited and the use is non-commercial. See: http:// creativecommons.org/licenses/by-nc/3.0/

\section{REFERENCES}

1. Fabbri G, Maggioni AP. A review of the epidemiological profile of patients with atrial fibrillation and heart failure. Expert Rev Cardiovasc Ther 2012;10:1133-40.

2. Menezes AR, Lavie CJ, DiNicolantonio JJ, et al. Atrial fibrillation in the 21st century: a current understanding of risk factors and primary prevention strategies. Mayo Clin Proc 2013;88:394-409.

3. Anderson JL, Halperin JL, Albert NM, et al. Management of patients with atrial fibrillation (compilation of 2006 ACCF/AHA/ESC and 2011 ACCF/AHA/HRS recommendations): a report of the American College of Cardiology/American Heart Association Task Force on Practice Guidelines. J Am Coll Cardiol 2013;61:1935-44.

4. Manlucu J, Brancato S, Lane C, et al. Contemporary approaches to persistent atrial fibrillation. Expert Rev Cardiovasc Ther 2012;10:1421-35.

5. Disertori M, Lombardi F, Barlera S, et al. Clinical characteristics of patients with asymptomatic recurrences of atrial fibrillation in the Gruppo Italiano per lo Studio della Sopravvivenza nell'Infarto Miocardico-Atrial Fibrillation (GISSI-AF) trial. Am Heart $J$ 2011;162:382-9.

6. Letsas KP, Weber R, Burkle G, et al. Pre-ablative predictors of atrial fibrillation recurrence following pulmonary vein isolation: the potentia role of inflammation. Europace 2009;11:158-63.

7. Liu T, Li L, Korantzopoulos P, et al. Meta-analysis of association between $\mathrm{C}$-reactive protein and immediate success of electrical cardioversion in persistent atrial fibrillation. Am J Cardiol 2008;101:1749-52.

8. Roberts WL, Sedrick R, Moulton L, et al. Evaluation of four automated high-sensitivity C-reactive protein methods: implications for clinical and epidemiological applications. Clin Chem 2000;46:461-8.

9. Celebi OO, Celebi S, Canbay A, et al. The effect of sinus rhythm restoration on high-sensitivity $\mathrm{C}$-reactive protein levels and their association with long-term atrial fibrillation recurrence after electrical cardioversion. Cardiology 2011;118:168-74.
10. Barassi A, Pezzilli $R$, Morselli-Labate AM, et al. Serum amyloid a and C-reactive protein independently predict the recurrences of atrial fibrillation after cardioversion in patients with preserved left ventricular function. Can J Cardiol 2012;28:537-41.

11. Hatzinikolaou-Kotsakou E, Tziakas D, Hotidis A, et al. Relation of $\mathrm{C}$-reactive protein to the first onset and the recurrence rate in lone atrial fibrillation. Am J Cardiol 2006;97:659-61.

12. Henningsen KM, Therkelsen SK, Bruunsgaard $\mathrm{H}$, et al. Prognostic impact of hs-CRP and IL-6 in patients with persistent atrial fibrillation treated with electrical cardioversion. Scand J Clin Lab Invest 2009;69:425-32.

13. Liu J, Fang PH, Dibs S, et al. High-sensitivity C-reactive protein as a predictor of atrial fibrillation recurrence after primary circumferential pulmonary vein isolation. Pacing Clin Electrophysiol 2011;34:398-406.

14. Lombardi F, Tundo F, Belletti S, et al. C-reactive protein but not atrial dysfunction predicts recurrences of atrial fibrillation after cardioversion in patients with preserved left ventricular function J Cardiovasc Med (Hagerstown) 2008;9:581-8.

15. Loricchio ML, Cianfrocca C, Pasceri V, et al. Relation of C-reactive protein to long-term risk of recurrence of atrial fibrillation after electrical cardioversion. Am J Cardiol 2007;99:1421-4.

16. Wazni $\mathrm{O}$, Martin $\mathrm{DO}$, Marrouche $\mathrm{NF}$, et al. $\mathrm{C}$ reactive protein concentration and recurrence of atrial fibrillation after electrical cardioversion. Heart 2005;91:1303-5.

17. Rizos I, Rigopoulos AG, Kalogeropoulos AS, et al. Hypertension and paroxysmal atrial fibrillation: a novel predictive role of high sensitivity C-reactive protein in cardioversion and long-term recurrence. J Hum Hypertens 2010:24:447-57.

18. Watanabe E, Arakawa T, Uchiyama T, et al. High-sensitivity C-reactive protein is predictive of successful cardioversion for atrial fibrillation and maintenance of sinus rhythm after conversion. Int $J$ Cardiol 2006;108:346-53.

19. Zarauza J, Rodriguez Lera MJ, Farinas Alvarez C, et al. Relationship between C-reactive protein level and early recurrence of atrial fibrillation after electrical cardioversion. Rev Esp Cardiol 2006:59:125-9.

20. Leeflang MM, Deeks JJ, Gatsonis C, et al. Systematic reviews of diagnostic test accuracy. Ann Intern Med 2008;149:889-97.

21. Arends LR, Hamza TH, van Houwelingen JC, et al. Bivariate random effects meta-analysis of ROC curves. Med Decis Making 2008;28:621-38.

22. Harbord RM, Whiting $P$, Sterne JA, et al. An empirical comparison of methods for meta-analysis of diagnostic accuracy showed hierarchical models are necessary. J Clin Epidemiol 2008;61:1095-103.

23. Higgins JP, Thompson SG, Deeks JJ, et al. Measuring inconsistency in meta-analyses. BMJ 2003;327:557-60.

24. Egger M, Davey Smith G, Schneider M, et al. Bias in meta-analysis detected by a simple, graphical test. BMJ 1997;315:629-34.

25. Buob A, Jung J, Siaplaouras S, et al. Discordant regulation of CRP and NT-proBNP plasma levels after electrical cardioversion of persistent atrial fibrillation. Pacing Clin Electrophysiol 2006;29:559-63.

26. Cosgrave J, Foley JB, Bahadur $\mathrm{K}$, et al. Inflammatory markers are not associated with outcomes following elective external cardioversion. Int J Cardiol 2006;110:373-7.

27. Ellinor PT, Low A, Patton KK, et al. C-Reactive protein in lone atrial fibrillation. Am J Cardiol 2006;97:1346-50.

28. Korantzopoulos P, Kolettis TM, Kountouris E, et al. Variation of inflammatory indexes after electrical cardioversion of persistent atrial fibrillation. Is there an association with early recurrence rates? Int J Clin Pract 2005:59:881-5.

29. Psychari SN, Chatzopoulos D, Iliodromitis EK, et al. C-reactive protein, interleukin 6 , and $\mathrm{N}$-terminal pro-brain natriuretic peptide following cardioversion of atrial fibrillation: is there a role of biomarkers in arrhythmia recurrence? Angiology 2011;62:310-16.

30. Komatsu T, Sato Y, Ozawa M, et al. Relationship between CHADS2 score and efficacy of antiarrhythmic drug therapy in patients with paroxysmal atrial fibrillation. Circ $J$ 2013;77:639-45.

31. Letsas KP, Efremidis M, Giannopoulos G, et al. CHADS2 and CHA2DS2-VASc scores as predictors of left atrial ablation outcomes for paroxysmal atrial fibrillation. Europace 2014;16:202-7.

32. Albert CM, Ma J, Rifai N, et al. Prospective study of C-reactive protein, homocysteine, and plasma lipid levels as predictors of sudden cardiac death. Circulation 2002;105:2595-9.

33. Parekh RS, Plantinga LC, Kao WH, et al. The association of sudden cardiac death with inflammation and other traditional risk factors. Kidney Int 2008;74:1335-42.

34. Streitner F, Kuschyk J, Veltmann C, et al. Role of proinflammatory markers and NT-proBNP in patients with an implantable cardioverterdefibrillator and an electrical storm. Cytokine 2009;47:166-72.

35. Korngold EC, Januzzi JL Jr, Gantzer ML, et al. Amino-terminal pro-B-type natriuretic peptide and high-sensitivity $\mathrm{C}$-reactive protein 
as predictors of sudden cardiac death among women. Circulation 2009;119:2868-76.

36. Engelmann MD, Svendsen JH. Inflammation in the genesis and perpetuation of atrial fibrillation. Eur Heart J 2005;26:2083-92.

37. Allessie M, Ausma J, Schotten U. Electrical, contractile and structural remodeling during atrial fibrillation. Cardiovasc Res 2002;54:230-46

38. Van Wagoner DR. Oxidative stress and inflammation in atrial fibrillation: role in pathogenesis and potential as a therapeutic target. J Cardiovasc Pharmacol 2008;52:306-13.
39. Moro C, Hernandez-Madrid A, Matia R. Non-antiarrhythmic drugs to prevent atrial fibrillation. Am J Cardiovasc Drugs 2010;10: $165-73$.

40. den Uijl DW, Delgado V, Tops LF, et al. Natriuretic peptide levels predict recurrence of atrial fibrillation after radiofrequency catheter ablation. Am Heart J 2011;161:197-203.

41. Tang Y, Yang H, Qu J. Relationship between brain natriuretic peptide and recurrence of atrial fibrillation after successful electrical cardioversion: a meta-analysis. J Int Med Res 2011;39: 1618-24. 